\title{
Latent inhibition from context-dependent retrieval of conflicting information
}

\author{
DENNIS C. WRIGHT, KARL D. SKALA, and KARL A. PEUSER \\ University of Missouri, Columbia, Missouri
}

\begin{abstract}
Rats were trained to lick and were tested for lick suppression to a tone in one apparatus/context. They were conditioned (tone-shock) in a different apparatus/context. Prior to conditioning, they received unreinforced preexposure to the tone (PCE) in the lick test box (Group A), in the conditioning box (Group X), or in a box different from both the test and conditioning boxes (Group B), or they received no PCE (Group N). In both Experiments 1 and 2, Group A was the only group to show latent inhibition (i.e., to show less conditioned lick suppression than did Group N). Context-dependent retrieval of "this CS signals nothing" information acquired during PCE reduces lick suppression when PCE and test contexts match, but not when PCE and conditioning contexts match.
\end{abstract}

When rats are given unreinforced presentations of a tone prior to the pairing of that tone with footshock, the amount of conditioned suppression of licking, which rats later show in the presence of the tone, is reduced (see, e.g., Lubow \& Siebert, 1969). This reduction in the manifestation of conditioned fear by unreinforced preconditioning exposure (PCE) to the stimulus has been labeled "latent inhibition" (LI). LI is often taken to be a demonstration that PCE reduces the rate/amount of conditioning that accrues to the stimulus when it later becomes a conditioned stimulus (CS) for footshock. As noted by Mackintosh (1983), PCE “will significantly retard subsequent conditioning [italics added] to that stimulus when it is paired with a reinforcer.... Most theorists have assumed that its associability with reinforcement must have declined" (p. 223).

The present experiments were designed to test the hypothesis that the reduction of conditioned suppression following PCE results from the retrieval during test of "this CS signals nothing" information learned during preexposure rather than from the failure of an animal to be conditioned to the preexposed CS. LI of conditioned lick suppression may result from retrieval of information during test that interferes with the retrieval of and/or manifestation of conditioned fear. A reduction in the associability of the CS during conditioning may be neither a necessary nor a sufficient condition for the production of LI.

The effects of PCE appear to be strikingly contextdependent (Anderson, O'Farrell, Formica, \& Caponigri, 1969; Bouton \& Bolles, 1979; Dexter \& Merrill, 1969; Hall \& Minor, 1984). LI appears to be maximal when PCE and conditioned suppression testing are conducted

\footnotetext{
We thank Donald H. Kausler and Ruth E. Wright for reviewing this manuscript and providing valuable comments. Requests for reprints should be sent to D. C. Wright, Department of Psychology, University of Missouri, Columbia, MO 65211.
}

in the same apparatus/context. If the "this CS signals nothing" information acquired during PCE is retrieved only in the context in which PCE was given, and if LI results from retrieval of such conflicting information during test, then PCE in any context other than the test context should produce no $\mathrm{LI}$.

This view is to be contrasted with the notion that PCE results in LI because of reduced conditioning. According to that notion, LI should be maximal when the PCE and conditioning contexts are matched, since that is the circumstance in which context-dependent retrieval of "this CS signals nothing" information could maximally interfere with conditioning.

\section{EXPERIMENT 1}

Three boxes that differed from one another visually, tactually, and in background sound and illumination level were used to provide different storage/retrieval contexts in this experiment. The context in which PCE was given was manipulated as the independent variable. One group of rats was given no PCE to provide a baseline measure from which to evaluate the occurrence of LI. The remaining groups received PCE in what was to be the test context (Group A), in what was to be the conditioning context (Group X), or in a context that was neither the test nor the conditioning context (Group B). All rats were lick trained and tested for suppression in the same context (Box A) that differed from the context in which all rats were fear conditioned (Box X). It was predicted that only Group A would show LI.

\section{Method}

Subjects. Forty male Sprague-Dawley rats (Sasco) served as subjects. All rats were individually housed in mesh-bottom cages, with a $12 / 12$ light/dark cycle and ad-lib food. Access to water was provided for $1 \mathrm{~h}$ each day immediately after the experimental session for that day. All experimental sessions were run during the 10-h time span between $1 \mathrm{~h}$ after lights on and $1 \mathrm{~h}$ before lights off. 
Apparatus. Box A, the lick train/test chamber, was constructed of Plexiglas and had inside dimensions of $40.6 \times 20.3 \times 30.5 \mathrm{~cm}$ and had a floor of $0.16-\mathrm{cm}$ mesh copper screen. A $0.64-\mathrm{cm}$ diameter hole centered in one $20.3-\mathrm{cm}$ end wall, $3.8 \mathrm{~cm}$ above the floor, permitted the lick tube to protrude $0.95 \mathrm{~cm}$ into the box. The lick tube was connected to a computer control system via a solid-state lickometer. The walls of Box A were painted with alternating 1.9-cm black and white stripes. Box X, the conditioning chamber, was $29.2 \times 22.2 \times 22.9 \mathrm{~cm}$ with three unpainted galvanized steel walls and a clear Plexiglas front. The shock grid floor was constructed of $0.32-\mathrm{cm}$ stainless steel rods mounted on 1.3-cm centers. In an additional preexposure box, Box B, all walls and the floor were constructed of Plexiglas that was painted flat black, and the box measured $29.8 \times 17.1 \times 25.4 \mathrm{~cm}$.

All three boxes had clear Plexiglas ceilings with an $8-\Omega, 1.2-\mathrm{cm}$ diameter speaker mounted at the center for delivery of an $86-\mathrm{dB}(\mathrm{A}), 3-\mathrm{kHz}$ sine wave tone CS. All three boxes were at different locations in the same room. The room was unlighted when Box B was in use and was lighted with fluorescent fixtures in the ceiling of the room when Box A was in use. The ambient noise level in both Box A and Box B was less than $60 \mathrm{~dB}(\mathrm{~A})$. Box $X$ was placed inside a sound-attenuation chamber with an exhaust fan that produced an ambient noise level of $66 \mathrm{~dB}(\mathrm{~A})$ within Box X and had a $25 \mathrm{~W}$ incandescent bulb centered $40.6 \mathrm{~cm}$ over the clear ceiling of Box X.

Procedure. One week after arriving from the supplier, the rats were given 7 days of handling and acclimation to the light/dark cycle and the 23-h water-deprivation schedule. Lick training, PCE, conditioning, and lick suppression testing sessions were then administered over 10 consecutive days as follows:

Days 1-5, lick training in Box A: During each daily session, the rats were allowed to lick for water for $300 \mathrm{sec}$ after the completion of the first 100 licks. The time required to complete the first 100 licks was recorded. The time required to reach Lick 100 on training Day 5 was used to assign 10 rats to each of four matched groups.

Days 6-8, PCE in Box A, B, or X: The rats in Groups A, B, and $X$ were presented five 10 -sec CSs delivered on a VT 60 -sec schedule during each daily 360 -sec session. All three sessions were given in Box A for Group A, in Box B for Group B, and in Box X for Group X. Group N rats spent three sessions in Box B, but no tones were presented. The lick tube was removed from Box A during the PCE sessions.

Day 9, conditioning in Box X: All rats received two tone-shock pairings (10-sec CS followed immediately by $1-\mathrm{sec}, 1-\mathrm{mA}$ scrambled footshock) during a single $360-$ sec session in Box X. The first pairing was delivered at $120 \mathrm{sec}$ and the second at $240 \mathrm{sec}$ after the start of the session.

Day 10, test in Box A: All rats received a single test for conditioned lick suppression in Box A. At the completion of Lick 100, the tone CS was turned on and remained on until the rat completed 10 additional licks or until $300 \mathrm{sec}$ had elapsed. The time from Lick 100 to Lick 110 (i.e., time to complete 10 licks in the presence of the CS) was recorded. Rats failing to complete those 10 licks were assigned a score of 300 , thus making nonparametric analyses appropriate for the test data.

\section{Results and Discussion}

Six rats were excluded from analysis of the test results. The data from 4 rats ( 1 in Group A, 1 in Group B, and 2 in Group $N$ ) were lost through equipment failure and 2 rats ( 1 in Group B, 1 in Group X) failed to complete 100 licks during the test. A Kruskal-Wallis one-way analysis of variance revealed no group difference in time to complete the first 100 licks $[H(3)=2.72]$.

The group median and mean times to complete 10 licks in the presence of the CS are shown in Table 1. As predicted, one-tailed Mann-Whitney tests revealed that Group A showed significantly less conditioned lick suppression than any other group (A vs. $\mathrm{B}, U=13$, $p<.025$; A vs. $\mathrm{X}, U=21, p<.05$; A vs. $\mathrm{N}, U=17$, $p<.05$ ) and that Groups $\mathrm{B}$ and $\mathrm{X}$ did not differ from
Table 1

Median and Mean Seconds from Lick 100 to Lick 110 in Experiments 1 and 2

\begin{tabular}{lcccrr}
\hline & \multicolumn{2}{c}{ Experiment 1} & & \multicolumn{2}{c}{ Experiment 2 } \\
\cline { 2 - 3 } \cline { 5 - 6 } Group & Median & Mean & & Median & Mean \\
\hline A & 114.08 & 153.33 & & 19.95 & 23.33 \\
X & 300.00 & 246.58 & & 137.22 & 203.47 \\
N & 300.00 & 257.25 & & 554.27 & 450.81 \\
B & 300.00 & 288.85 & & \\
\hline
\end{tabular}

Note-Group B was not run in Experiment 2.

Group $\mathrm{N}$ or from one another (B vs. $\mathrm{N}, U=30$; $\mathrm{X}$ vs. $\mathrm{N}, U=33$; B vs. $\mathrm{X}, U=31$ ). (Parametric analyses of the data were also performed. Analysis of variance, followed by independent $t$ tests, yielded an identical pattern of statistical significance.) Thus, Group A was the only group to manifest LI, providing clear evidence that under the conditions of this experiment, context-dependent retrieval during test of information acquired during PCE is a sufficient condition for the production of LI.

It is reasonable to argue that PCE should maximally reduce conditioning when the conditions for the retrieval of PCE at the time of conditioning are optimized (i.e., when the PCE and conditioning contexts match). The failure of Group $X$ to show $\mathrm{LI}$ in comparison to Group $\mathrm{N}$ provides evidence that if conditioning is reduced by PCE, it is not a sufficient condition for the production of $\mathrm{LI}$.

\section{EXPERIMENT 2}

Experiment 2 is essentially a replication of Experiment 1 , with some changes in procedure. First, the number of preexposures to the tone CS was increased from 15 to 30 . It can be argued that although retrieval of conflicting information can interfere with the manifestation of fear in test following relatively few PCEs, interference with conditioning may require more PCEs. Second, the test cutoff time was increased from 300 to $900 \mathrm{sec}$. Whereas the short test cutoff in Experiment 1 strengthens the argument that Group A differed from the remaining groups, it weakens the argument that Group X failed to differ from Group N. Finally, financial considerations dictated the elimination of Group B and a reduction in $n s$.

\section{Method}

Subjects. Nineteen male Sprague-Dawley rats (Sasco) served as subjects under conditions identical to those described for Experiment 1.

Apparatus. The apparatus used was the same as that used in Experiment 1.

Procedure. The procedures were identical to those of Experiment 1, with the following exceptions:

Following five lick training sessions in Box A, time to Lick 100 was used to assign 7 rats to Group A, 7 to Group X, and 5 to Group N.

During each of the three 480-sec PCE sessions, the rats in Groups $A$ and $X$ were presented 10 10-sec CSs delivered on a VT 44-sec schedule. As before, the PCE sessions were given in Box A for Group A, in Box X for Group X, and in Box B with no tones presented for Group N.

The conditioning session was also $480 \mathrm{sec}$ in duration, with the first pairing at $120 \mathrm{sec}$ and the second at $360 \mathrm{sec}$ after the start of the session. 
The day following the single conditioning session in Box $X$, the rats were tested for conditioned lick suppression in Box A, as in Experiment 1 , but the maximum time allowed to complete 10 licks in the presence of the CS was $900 \mathrm{sec}$.

\section{Results and Discussion}

One rat (Group $\mathrm{X}$ ) failed to complete 100 licks during test and was eliminated from the analysis of results. The Kruskal-Wallis analysis of time to complete the first 100 licks during the test again revealed no group difference $[H(2)=5.09]$.

All animals completed 10 licks in the presence of the CS. The group median and mean times to complete 10 licks in the presence of the CS are shown in Table 1. As predicted, one-tailed Mann-Whitney tests revealed that Group A showed significantly less suppression than did Group X $(U=17, p=.026)$ and Group $\mathrm{N}(U=0$, $p<.001)$, and that Groups $\mathrm{N}$ and $\mathrm{X}$ did not differ from each other $(U=7, p=.089)$. Again, parametric analyses yielded an identical pattern of statistical significance. As in Experiment 1, Group A was the only group to show evidence of LI.

The major pretest procedural difference between Experiments 1 and 2 was a doubling of the number of PCEs. As expected (e.g., Lantz, 1973), increasing the number of PCEs in Experiment 2 appears to dramatically increase the amount of LI shown by Group A, as seems evident from examining the Group A scores for Experiments 1 and 2. These rats had median scores of 114.08 in Experiment 1 and 19.95 in Experiment 2.

The apparent change in median and mean scores seemed sufficiently interesting to warrant preliminary examination by unorthodox cross-experiment comparisons. Since a 300-sec cutoff had been used in Experiment 1, Experiment 2 scores that exceeded $300 \mathrm{sec}$ were analyzed as scores of 300, as they had been in Experiment 1. The Group A Experiment 1 scores differed from Group A Experiment 2 scores $(U=9, p=.01)$ but Experiment 1 and 2 scores did not differ reliably for Group $\mathrm{X}(U=17)$ or Group $N(U=17)$. Thus, doubling the number of PCEs in Experiment 2 led to further reductions in lick suppression for Group A, but had no apparent effect on Group X.

\section{GENERAL DISCUSSION}

The data reported here confirm earlier reports (Anderson et al., 1969; Bouton \& Bolles, 1979; Dexter \& Merrill, 1969; Hall \& Minor, 1984) that PCE reduces conditioned suppression maximally when the PCE and test contexts match. In addition, the data provide evidence that rats run with a match between PCE and conditioning contexts, a procedure designed to maximally reduce conditioning, show neither more nor less conditioned suppression than rats given no PCE. In short, PCE produces LI only if testing occurs in the context in which PCE was given. The simplest explanation of that result appears to be that the retrieval during test of "this CS signals nothing" information acquired during PCE interferes with the retrieval of and/or manifestation of conditioned fear. Moreover, since neither Group X nor Group B showed LI, the retrieval of interfering information during test appears to be context-dependent. The group for which PCE would be predicted to maximally "retard subsequent conditioning," Group X, did not show LI. Thus, although this paradigm provides evidence that the absence of LI can result from a context-dependent retrieval failure, it provides no evidence that $\mathrm{LI}$ can result from a failure to store conditioned fear.

It should be noted that Group X showed a trend for PCE-produced reduction of conditioned suppression in Experiment 2. Lubow, Rifkin, and Alek (1976) and Channell and Hall (1981) gave rats prolonged preexposure to stimuli that were to be the discriminanda in a discrimination learning paradigm. Preexposure in the apparatus/context in which the task was to be learned retarded subsequent acquisition. It may well be the case that extensive PCE in the conditioning context can result in reduced conditioning. Alternatively, it may be the case that PCE "overtraining" could result in reduced context dependencey for retrieval of interfering "this CS signals nothing" information in much the same manner that overtraining reduces state-dependent retrieval (Bliss, 1972; Iwahara \& Noguchi, 1972; Modrow \& Bliss, 1979). It remains for future research to determine whether extensive PCE in the conditioning context will produce LI and whether any such LI results from reduced conditioning or from cross-context retrieval of interfering information at test.

\section{REFERENCES}

Anderson, D. C., O'Farrell, T., Formica, R., \& CaPonigri, V. (1969). Preconditioning CS exposure: Variation in place of conditioning and of presentation. Psychonomic Science, 15, 54-55.

BLIss, D. K. (1972). Dissociated learning and state-dependent retention induced by pentobarbital in rhesus monkeys. Journal of Comparative \& Physiological Psychology, 84, 149-161.

Bouton, M. E., \& Bolles, R. C. (1979). Contextual control of the extinction of conditioned fear. Learning \& Motivation, 10, 445-466.

Channell, S., \& Hall, G. (1981). Facilitation and retardation of discrimination learning after exposure to the stimuli. Journal of Experimental Psychology: Animal Behavior Processes, 7, 437-446.

DEXTER, W. R., \& MERRILL, H. K. (1969). Role of contextual discrimination in fear conditioning. Journal of Comparative \& Physiological Psychology, 69, 677-681.

HALl, G., \& MiNOR, H. (1984). A search for context-stimulus associations in latent inhibition. Quarterly Journal of Experimental Psychology, 36B, 145-169.

IwAHARA, S., \& NoGUCHI, S. (1972). Drug state dependency as a function of overtraining in rats. Japanese Psychological Research, 14, 141-144.

LANTZ, A. E. (1973). Effect of number of trials, interstimulus interval, and dishabituation during CS habituation on subsequent conditioning in a CER paradigm. Animal Learning \& Behavior, 1, 273-277.

Lubow, R. E., Rifkin, B., \& ALEK, M. (1976). The context effect: The relationship between stimulus preexposure and environmental preexposure determines subsequent learning. Journal of Experimental Psychology: Animal Behavior Processes, 2, 38-47.

Lubow, R. E., \& SIEBERT, L. (1969). Latent inhibition within the CER paradigm. Journal of Comparative \& Physiological Psychology, 68, 136-138.

MaCKIntosh, N. J. (1983). Conditioning and associative learning. New York: Oxford University Press.

Modrow, H. E., \& Bliss, D. K. (1979). Electrophysiological correlates of state-dependent learning. Physiological Psychology, 7, 259-262.

(Manuscript received for publication October 17, 1985.) 